\title{
Note sur la spirocercose canine dans la république de Haute-Volta
}

\author{
par M. G. REGNOULT, J. DUBOIS ef R. FREDET
}

La présente note a pour objet d'attirer l'attention sur la fréquence dans la région de BoboDioulasso (République de Haute-Volta) de l'affection vermidienne connue sous le nom de Spirocercose canine, due à Spirocerca sanguinolento.

Nous avons présentement observé une vingtaine de cas, aboutissant tous généralement à la mort, la découverte de l'affection étant faite à l'autopsie mais les signes cliniques ayant une relative constance et étant susceptibles souvent d'être confondus avec ceux de la rage.

\section{$*^{*} *$}

Spirocerca sanguinolenta (Rudolfi. 1819) responsable de l'affection est un nématode de la famille des Filariidae.

- Le mâle, de couleur rouge sang, comme la femelle, mesure de 30 à $55 \mathrm{~mm}$ de long sur $760 \mu$ de large, l'œsophage est long de $5.8 \mathrm{~mm}$, le spicule gauche mesure $2,45 \mathrm{~mm}$ et le droit $750 \mu$ seulement, la queve est enroulée ventralement sur elle-même.

- La femelle mesure de 55 à $80 \mathrm{~mm}$ de long sur $1,15 \mathrm{~mm}$ de large, l'œsophage a une tongueur de $7 \mathrm{~mm}$ environ, la vulve est en avant ou en arrière de l'extrémité postérieure de l'œsophage et à 2 à $4 \mathrm{~mm}$ de l'extrémité céphalique, la queve est légèrement recourbée dorsalement.

L'extrémité céphalique des deux genres est pourvue d'une bouche entourée de six petites papilles, le vestibule a une profondeur de 90 à $100 \mu$ et une largeur égale réduite postérieurement.

Les œufs sont cylindriques et mesurent de 30 ì $37,5 \mu$ de long sur 11 à 15 de large.

La biologie de ce Nématode est connue. Les œufs, souvent embryonnés, trouvés dans les excréments de l'hôte définitif (chien, loup, cha-

Reçu pour publication : mars 1961.

Rev. Elev. Méd. vét. Pays trop. 1961, 14, nº 2. cal, renard) sont ingérés par des coléoptères coprophages, hôtes intermédiaires, chè lesquels l'éclosion a lieu dans le tube digestif; la larve au troisième stade s'y enkyste. L'hôte définitif ingère cette larve en même temps que le coléoptère ou qu'un vertébré insectivore, hôte accidentel du parasite (crapaud, lézard, serpent, poulet, etc...). Chez l'hôte définitif, la larve au troisième stade quitte son kyste, traverse la paroi de l'œsophage ou de l'estomac et effectue des migrations compliquées avant d'aller se localiser dans la paroi de l'œsophage ou de l'aorte, au quatrième stade et à l'état adulte,' le parasite y provoque la formation d'un nodule. Ce nodule est perforé d'un orifice par lequel les œufs sont émis dans la lumière du tube digestif ou dans la circulation générale, seion la localisation.

La spirocercose canine est une affection cosmopolite, principalement répandue dans les pays chauds : pourtour méditerranéen, Japon, Chine, Brésil, Madagascar, Afrique occidentale (dont la République de Haute-Volta qui fait l'objet de la présente publication).

Le diagnostic de l'affection est difficile. La découverte des œufs et des embryons dans les excréments, les expectorations ef le sang permet seule de suspecter la maladie. Les signes observés peuvent faire penser selon les cas à une gastrite aiguë, une filariose, une bronchite chronique, la tuberculose ou la rage. Nous attirons particulièrement l'attention sur ce dernier point, en Afrique occidentale, où la rage est endémique. En effet, dans la spirocercose, l'animal présente souvent des troubles nerveux extrêmement graves qui simulent des accès de, rage : crises d'excitation, de fureur, tendance à mordre et autres phénomènes d'irritabilité ; il est à noter également la grande fréquence du ptyalisme, de la dysphagie, du vomissement, de l'anorexie. Si les nodules siègent au niveau de l'aorte, l'animal peut présenter des crises de dyspnée, de 
suffocation, d'angoisse, parfois des syncopes. Quand les nématodes sont fixés en région pulmonaire on peut observer des phénomènes de bronchite avec inspiration brève et bruyante, toux accompagnée de nausées, vomissements hémorragiques.

$\mathrm{Si}$, d'après les signes cliniques on pense à la spirocercose, il faut être très réservé sur le pronostic car on ne connaît pas de médicament susceptible d'atteindre à coup sûr les parasites à l'intérieur des nodules. On peut seulement administrer une thérapeutique symptomatique. Le plus souvent, comme nous l'avons dit, l'affection est découverte à l'autopsie, l'issue de la maladie étant considérée comme fatale.

Les nodules provoqués par Spirocerca sanguinolenta se rencontrent surtout dans la portion thoracique de l'œsophage. Ces nodules, en petit nombre, ont un volume variant de celui d'un pois à celui d'un œuf de poulette, mais peuvent parfois atteindre la taille du poing. Leur consistance est ferme, ils sont globuleux ou ovoïdes et présentent une perforation à leur sommet. A l'intérieur du nodule se trouve un pus séro-sanguinolent parmi lequel vivent les parasites, enroulés sur eux-mêmes, en un paquet de plusieurs unités à quelques dizaines d'individus. Les nodules aortiques sont plus durs, plus épais et plus nombreux, quand ils existent, que les nodules œsophagiens, ils sont plus petits. Les nodules pulmonaires sont blanchâtres, gros, arrondis et consistants, creusés de cavités où sont logés les parasites.

La prophylaxie de l'affection consiste principalement à empêcher les chiens de manger les insectes, les lézards et les tripes de volailles.

\section{OBSERVATIONS}

\section{Observation I. - Juillet-septembre 1960}

Chienne Boxer bringée âgée de 5 ans, appartenant à M. L., transporteur à Bobo-Dioulasso.

- Durant l'absence de son maître, parti en congé, la chienne a présenté en juillet 1960 des signes qui ont passé pour une intoxication alimentaire (violents vomissements, nausées, abattement et anorexie). Après un traitement symptomatique, l'animal, soigné à la clinique de Ouagadougou, a retrouvé une meilleure santé. Quelques semaines plus tard, les signes d'une violente conjonctivite et de congestion oculaire apparaissaient. On administra de l'hydrocortisone, ce qui amena une très légère amélioration ; dans la crainte d'une rickettsiose, on prescrivit de la Tifomycine : sans résultat.

C'est en fin septembre 1960 que la chienne nous fut présentée à Bobo-Dioulasso pour la première fois. L'animal manquait d'appétit ef était pratiquement aveugle : l'œil droit, entièrement opacifié, n'avait plus aucune vision même celle de la lumière, - quant à l'œil gauche, il présentait une kératite opacifiante à son début. Abandonnant les précédents traitements qui n'avaient, donné aucun résultat, nous instituons une thérapeutique vitaminique intensive (vitamines A, C et D à hautes doses), nous saupoudrons la conjonctive avec du calomel au sucre. Au bout du quatrième jour nous observons une légère amélioration qui permet de procéder àun examen ophtalmoscopique complet. nous observons une iridocyclite avec forte rétinite. Notre pronostic est très réservé quant d̀ l'évolution de la cécité que nous avons toutes raisons de penser devoir s'accentuer. Au cours d'une prise de sang pour recherche de rickettsiose, nous remarquons la couleur anormalement claire du sang et son défaut d'agglutination rapide, le temps de saignement est également notablement augmenté. Nous laissons l'animal à son traitement vitaminique. Quelques temps après nous apprenons que la chienne est à nouveau prise de nausées, qu'elle a de la peine à s'alimenter, éprouvant de la gêne à déglutir et bavant abondamment. Partant en tournée, nous ne pouvons revoir l'animal avant notre départ et c'est d̀ notre retour que nous apprenons qu'ayant été trouvé mort un matin après avoir présenté de l'épistaxis, il a été porté au Service de l'élevage où son autopsie a été pratiquée.

Trois nodules à Spirocerca sanguinolenta étaient implantés sur l'œsophage en position médiastinale dont deux à l'entrée de l'estomac. La compression effectuée par les nodules sur les gros vaisseaux de l'entrée du cou pouvait-elle expliquer les troubles oculaires?

\section{Observation II. - Août 1960}

Chien bâtard de 3 ans, appartenant à M. B., à Bobo-Dioulasso. - Autopsié à la clinique de Bobo-Dioulasso après avoir présenté des signes 
rabiformes d'excitation, d'irritabilité, du ptyalisme et de la dysphagie. Quatre nodules à Spirocerca sanguinolenta, de la grosseur d'une noix chacun, siégeaient au niveau du tiers moyen de la portion céphalique de l'œsophage.

\section{Observation III. - Février 1960}

Jeune chien berger allemand, appartenant à M. L., entrepreneur à Bobo-Dioulasso.

- Nous sommes appelés le matin du 11 février 1960 vers 11 heures par le propriétaire dont le chien «fait une crise » subitement. Nous trouvons ce chien, âgé de 4 mois, en décubitus latéral complet, mouvements de pédalage, forte excitation ef ptyalisme.

Ecartant le tétanos pour absence d'opisthotonos caractéristique, nous pensons à un coup de soleil et de chaleur. Nous envisageons momentanément la possibilité d'une réaction éventuelle de ce jeune chien à une vaccination antirabique très récente, datant de 8 jours.

Après mise à l'ombre, aspersion de la tête, administration de tonicardiaques, l'état du chien s'améliore légèrement, puis empire dans l'aprèsmidi. Un vomissement sanguinolent nous permet un diagnostic presque certain de spirocercose.

L'animal meurt dans la nuit et le lendemain matin l'autopsie confirme la suspicion : plusieurs nodules spiruriens sur l'œsophage médiastinal et 1 nodule duodénal. C'est le plus jeune animal rencontré atteint de cette affection.

\section{Observation IV. - Octobre 1960}

Chienne Boxer fauve 3 ans, appartenant à $M$. C. Société Etaperu à Bobo-Dioulasso.

- La chienne est amenée au Service à 9 heures pour raideur et douleur lombaire avec gêne dans la locomotion, ce qui nous fait penser à une myosite par refroidissement (saison d'hivernage).

Nous sommes rappelés à 13 heures au domicile du propriétaire. L'animal a présenté une crise d'excitation alarmante et subite. Administration de Largactyl et de toni-cardiaques. Grande faiblesse et irrégularité du: pouls, plyalisme.

La chienne meurt à 14 heures. A l'autopsie : nodules spiruriens sur l'œesophage et l'estomac, l'éclatement d'un nodule a provoqué une hémorragie interne.

Commémoratifs: jusque là en excellente scnté ef en très bon éfat d'entretien, cette chienne a eu 8 jours avant un vomissement très légèrement sanguinolent.

\section{Observation V. - Février 1960}

Chien berger allemand, appartenant à M. S., Compagnie Transafricaine à Bobo-Dioulasso.

- Le chien, adulte, est présenté au Service pour excitation ef changement de caractère, un ou deux vomissements légèrement hémorragiques : forte suspicion de rage.

Le chien meurt quelques heures après l'examen.

\section{A l'autopsie : nodules spiruriens}

Des prélèvements sont envoyés au Laboratoire de l'Elevage «G. CURASSON » à Dakar (bulbe rachidien et corne d'ammon) qui répond: roge positive.

Dans ce cas nous avons observé concomitance de spirurose et de rage confirmée, ce qui montre de quelles précautions il convient de s'entourer.

Plusieurs autres animaux trouvés porteurs de nodules spiruriens à l'autopsie et présentés à la clinique au cours des années 1958 ef 1959 pour des crises nerveuses accompagnées de vomissement, vu la suspicion de rage qui pesait sur eux ont fait l'objet de prélèvements expédiés au Laboratoire de l'Elevage de Dakar. Le diagnostic fut «rage positive ». L'association sur un même animal des deux affections n'est donc pas rare et la découverte de nodules spiruriens ne doit pas faire présumer l'absence de rage.

\section{RÉSUMÉ}

La spirocercose canine, due à $S$. sanguinolento présente des symptômes très divers, dominés par une excitation presque constante, rabiforme, avec issue rapide vers la mort.

Le diagnostic clinique, très réservé en raison de sa difficulté, est étayé par la présence de vomissements sanguinolents, d'épistaxis légers, parfois de moelena, signant la rupture d'un nodule. 
Une autopsie rapide peut omettre des lésions de Spirurose souvent discrètes. L'attention a été attirée sur cette affection lors d'autopsies pratiquées en 1958, à Bobo-Dioulasso, par le Chef de Circonscription d'Elevage; depuis lors, la recherche systématique des nodules spiruriens, lors des autopsies de canidés, a révélé la grande fréquence de la Spirocercose canine dans l'Ouest-Volta.

\section{SUMMARY}

\section{Note on Canine Spirocercosis in the Republic of the Upper Volta}

Canine Spirocercosis, due to Spirocerca sanguinolata, is manifested by a great variety of symptoms but dominated almost constantly by excitement as in rabies, particularly just prior to death. Clinical diagnosis present some difficulty, but is indicated by blood-stained vomit, light epistaxis and accasionally moelena (blood in faeces?) which signifies the rupture of a nodule. If a hasty post-mortem is made the lesions of spirocercosis may be overlooked. A systematic examination for nodules has demonstrated a high incidence of canine spirocercosis in West Volta.

\section{RESUMEN}

Nota sobre la espirocercosis canina en la República de Alto Volta

La espirocercosis canina, debida a Espirocerca sanguinea se manifiesta por unos sintomas, muy diversos, entre los que domina una excitación casi constante, semejante a la que se observa en la rabia, que terminan con la muerte.

El diagnóstico clínico, reservado en razón de su dificultad, es basado en los vómitos sanguinolentos, epístas is ligeras, presencia de sangre en los excrementos, que indican la roptura de un nódulo.

Una autopsia rápida puede no descubrir lesiones de espirocercosis a menudo discretas. Si se buscan de $m$ anera sistemática los nódulos, se aprecia la gran frecuencia de la espirocercosis canina en el œste del país.

\section{BIBLIOGRAPHIE}

\section{NEVEU LEMAIRE. - Traité d'Helminthologie Médicale ef Vétérinaire.}

HULIN (P.), ROBINET (A. H.), RIVIERE (R.). -

Un cas de Spirocercose Canine. Bull. El. Ind.
Anim. A. O. F. (1949), 11 (4), 38.

THOROLD (P. W.) et HOLMES (C. R.). - L'infestation des chiens à Spirocerca lupi au Kenya.. Bull. Epiz. Dis. Afr. (1956), IV, (4), 321. 